\title{
La interacción de la luz con moléculas
}

\author{
Bárbara Paulet Domínguez Capitaine, Héctor Hugo Cerecedo \\ Núñez, Patricia Padilla Sosa y Josué Ismael García Ramírez
}

\begin{abstract}
Resumen
Con el fin de comprender los diferentes mecanismos que existen entre la interacción de la luz y las moléculas orgánicas e inorgánicas, en este artículo se describen, de forma general, los procesos que una molécula puede seguir al ser excitada por radiación luminosa. Esto, a la vez, ayuda a interpretar fenómenos que se pueden observar en un laboratorio, o en algo más cercano a nosotros, como la naturaleza. Para realizar esta descripción analizamos las clasificaciones ya existentes de estos mecanismos. La interacción de la luz puede establecerse a través de distintos procesos: fotofísicos, fotoquímicos, radiativos y no radiativos. De tal manera que la clasificación dependerá del tipo de molécula y de la naturaleza de los enlaces (uniones o conexiones) químicos implicados. Por tal motivo, este artículo está enfocado en describir dichos procesos, al ejemplificar los fenómenos con los que se relacionan. Se busca que el lector comprenda, de forma general, los distintos mecanismos de interacción de la luz con moléculas.
\end{abstract}

Palabras clave: interacción radiación-materia, luz, moléculas, procesos fotoquímicos, procesos fotofísicos, procesos radiativos, procesos no radiativos.

\section{THE INTERACTION OF LIGHT WITH MOLECULES}

\begin{abstract}
In order to understand the different phenomena that exist between light interaction and organic or inorganic molecules, this article describes, in general, the processes that a molecule can follow when excited by light radiation. This, in turn, helps to interpret phenomena observed in a laboratory, or something closer to us, such as nature. To make this description, we analyze the existing classifications of these mechanisms. The interaction of light can be established through different processes: photophysical, photochemical, radiative, and non-radiative. Thus, the classification will depend on the type of molecule and the nature of the chemical bonds (connections). For this reason, this article focuses on describing such processes, exemplifying the phenomena to which they relate. The aim is that the reader can obtain a general understanding of the different mechanisms of light and molecules interaction.
\end{abstract}

Keywords: radiation-matter interaction, light, molecules, photochemical processes, photophysical processes, radiative processes, non-radiative processes.

Recepción: 03/06/2018. Aceptación: 20/05/2020 Dol: http://doi.org/10.22201/cuaieed.16076079e.2020.21.5.1 
"La interacción de la luz con moléculas" Bárbara Paulet Domínguez Capitaine, Héctor Hugo Cerecedo Núñez, Patricia Padilla Sosa y Josué Ismael García Ramírez

Vol. 21, Núm. 5, septiembre-octubre 2020 Revista Digital Universitaria

Bárbara Paulet Domínguez Capitaine barbara.pdc1@gmail.com

Ingeniera química por la Universidad Veracruzana, en 2019. Actualmente es estudiante de la Maestría en Ingeniería de Corrosión en el Instituto de Ingeniería de la Universidad Veracruzana.

Héctor Hugo Cerecedo Núñez

hcerecedo@uv.mx www.uv.mx/loa orcid.org/0000-0001-8132-7272 www.researchgate.net/profile/H_Cerecedo-Nunez

Google Scholar Citations: H. H. Cerecedo-Núñez

Licenciatura en Física (Facultad de Física, Universidad Veracruzana), Posdoctorado en el Instituto Tecnológico de Virginia (Blacksburg, VA, USA). Actualmente es investigador y profesor en la Facultad de Física de la Universidad Veracruzana. Desarrolla actividades en el área de Óptica Aplicada, en diversos proyectos relacionados con fotónica, biofotónica, sensores y monitoreo de parámetros físicos con luz. Cofundador del Laboratorio de Óptica Aplicada de la misma entidad. Ha participado en varios proyectos de investigación y académicos, en los cuales se han logrado obtener recursos económicos para infraestructura de laboratorios de enseñanza e investigación. Ha publicado artículos en revistas indizadas, en memorias en extenso y capítulos en libros (arbitrados, y relacionados con física y óptica aplicada).

\section{Patricia Padilla Sosa}

ppadilla@uv.mx

Licenciatura en física, con Doctorado en Ciencias con especialidad en Óptica, actualmente académica de la Universidad Veracruzana, Facultad de Física. Coordinadora y colaboradora del cuerpo académico de óptica aplicada y materia condensada blanda.

Josué Ismael García Ramírez

jonas9316@hotmail.com

Licenciado en física por la Universidad Veracruzana, en 2018. Actualmente es estudiante de la Maestría en Ciencias en Micro y Nanosistemas del Centro de Investigación en Micro y Nanotecnología (MICRONA) de la misma universidad. 


\section{Introducción}

La luz es radiación electromagnética comprendida en un rango (espectro visible) en el cual nuestros ojos son capaces de percibirla (ver figura 1). La luz es capaz de transportar energía, por lo que también tiene un papel fundamental en todos aquellos elementos con los que interactúa. Por ejemplo, la interacción de la luz con los seres vivos da lugar a muchos procesos vitales, como la fotosíntesis, síntesis de vitaminas, entre otros. También podemos observar cómo la materia, en general, puede absorber, transmitir, reflejar, o incluso, "transformar" la luz. Estos hechos suceden bajo determinadas condiciones; para ello, la energía de la radiación electromagnética debe "coincidir" con la energía que hay en los enlaces (uniones o conexiones) de las moléculas. De otra forma, podría no haber efecto alguno, o éste podrá ser ineficiente.

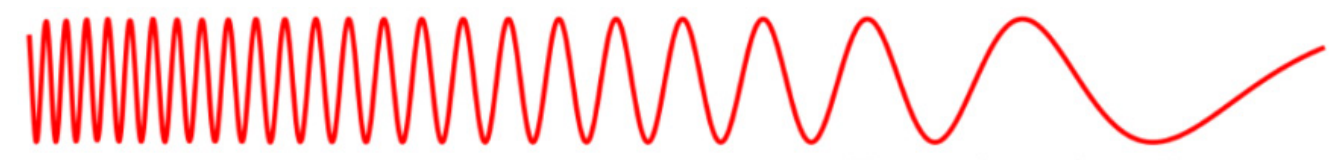

Figura 1. Espectro electromagnético [adaptación de Philip Ronan, Gringer (2008)].

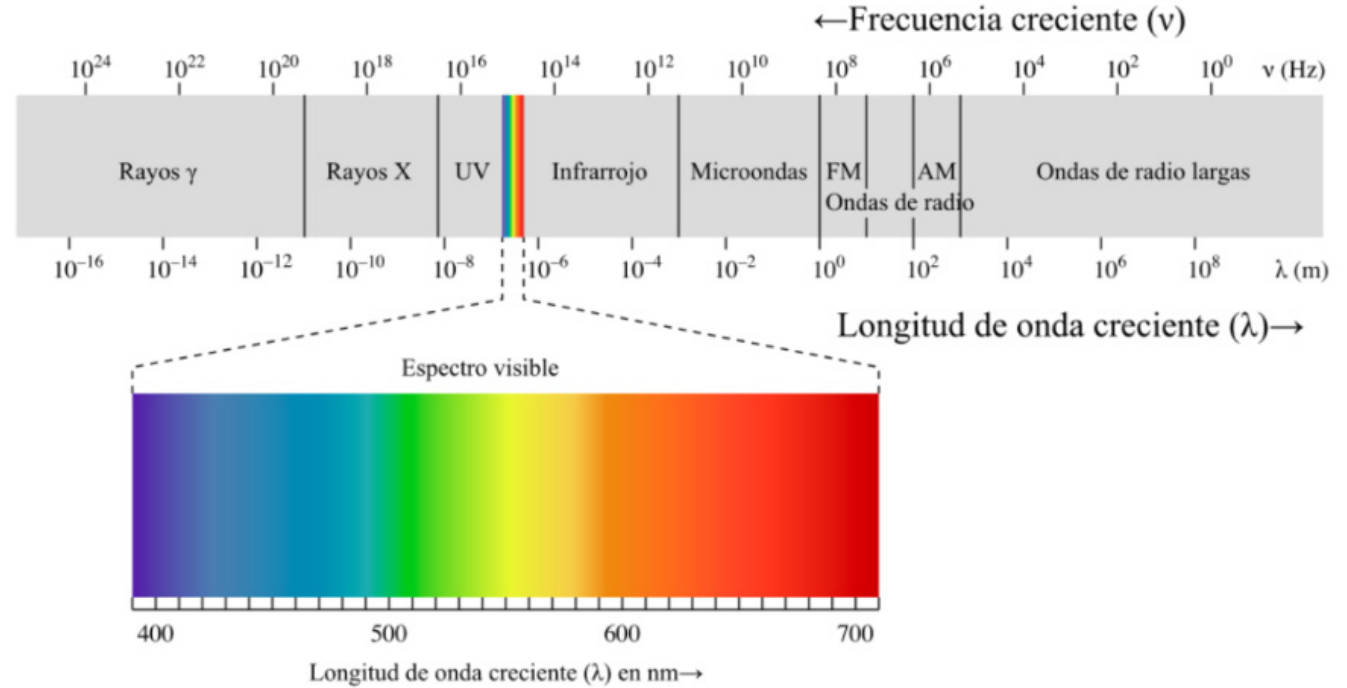

Las moléculas estables (no alteradas) suelen tener sus enlaces bajo cierto estado de energía (energía base o estado base, descritos como: s, p, d, f, ver figura 2a). Las moléculas que absorben radiación electromagnética conducen a la excitación de sus enlaces moleculares (estados de energía), llevándola a un estado con mayor energía (excitado), un ejemplo de ello se representa en la figura 2b. Entonces, lo que suceda entre la luz y una molécula, dependerá del tipo de enlace que tengan, y del tipo de radiación que incide sobre ellas. Así, bajo condiciones de excitación, una molécula tampoco puede permanecer por mucho tiempo en un estado excitado (a menos que continúe recibiendo energía), después, ésta buscará diferentes caminos para perder el exceso de energía adquirido (proceso de desexcitación, ver figura 3). Entre estos caminos podemos encontrar dos clasificaciones: procesos fotofísicos y fotoquímicos, y procesos radiativos y no radiativos. 
Figura 2. Representaciones de: a) orbitales básicos [Adaptación de la fuente: Gela Irualia, 2007]

y b) orbitales moleculares

(enlaces) [adaptación de la fuente: Chemical Bonding, Chemical Formulas, 2020], Ios cuales pueden mezclarse al adquirir energía, o bien, al pasar de un estado básico a un estado excitado. Un orbital atómico es la región del espacio donde se mueven los electrones.

Figura 3. Ejemplo de transiciones de energía en una molécula, a través de sus distintos niveles de energía. Representación en diagrama de niveles de energía (diagrama de Jablonski) para la excitación de una molécula de Helio y Neón (adaptación de Xu Panda, 2017).

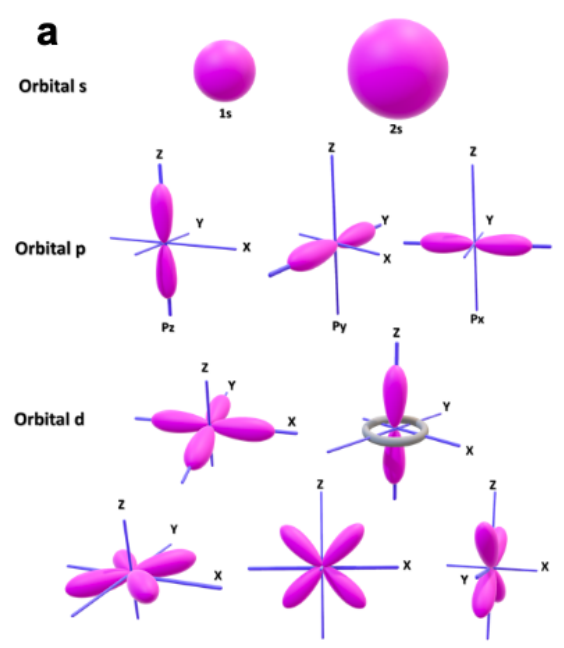

b
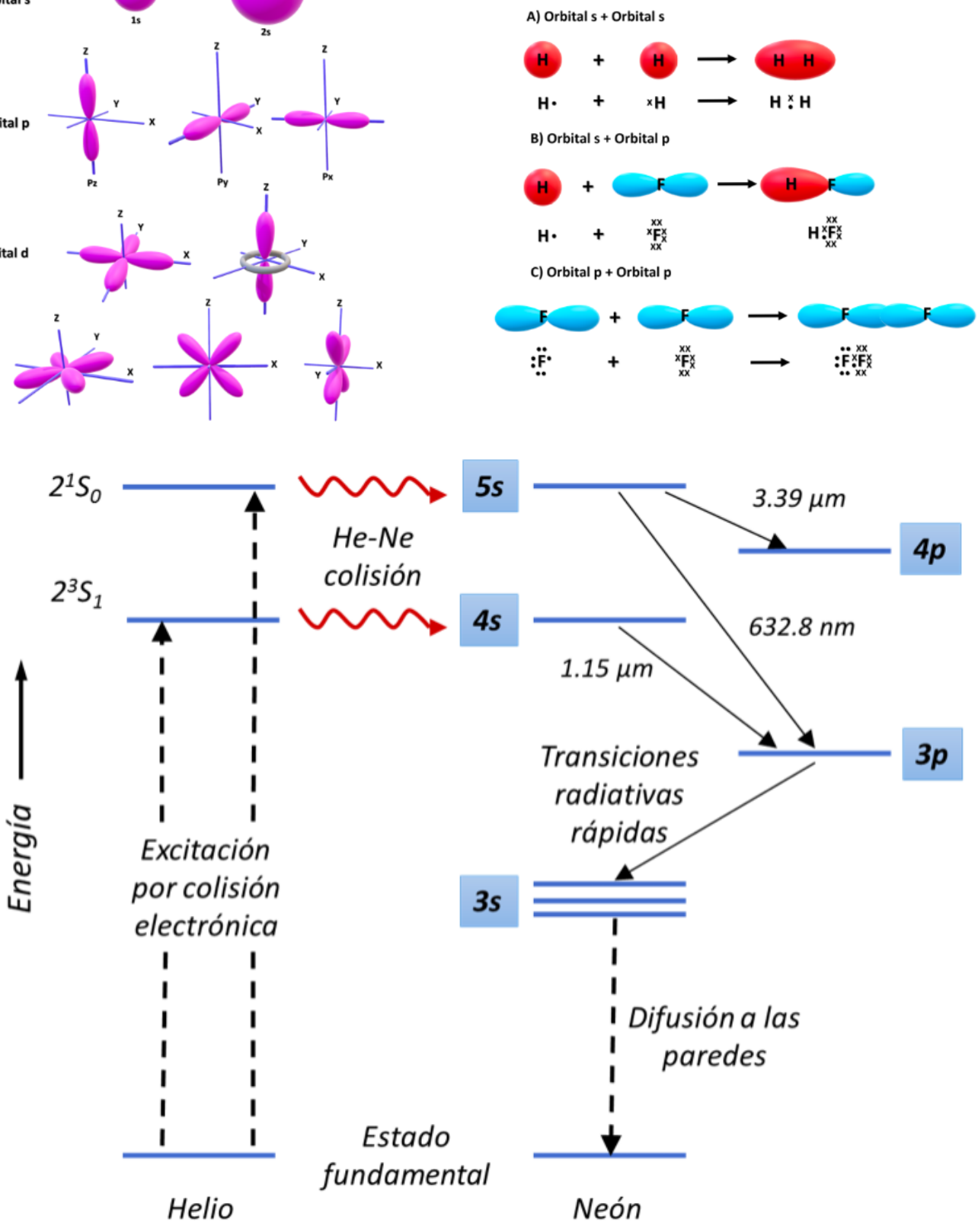

Los procesos fotofísicos no afectan la naturaleza química de la molécula y la forma de desexcitación ocurre por fenómenos como la emisión de radiación electromagnética (fluorescencia y fosforescencia) o transferencia de calor. Mientras que los procesos fotoquímicos generan estados altamente excitados que conllevan a una gran reacción química (ver figura 4). Por lo tanto, se puede llevar a cabo un cambio químico mediante luz, siempre y cuando ésta sea absorbida adecuadamente por alguna de las moléculas involucradas. En general, la modificación química es la gran diferencia entre ambos procesos. 
Figura 4. Procesos fotoquímicos y fotofísicos

(adaptación de Lorente, 2003)
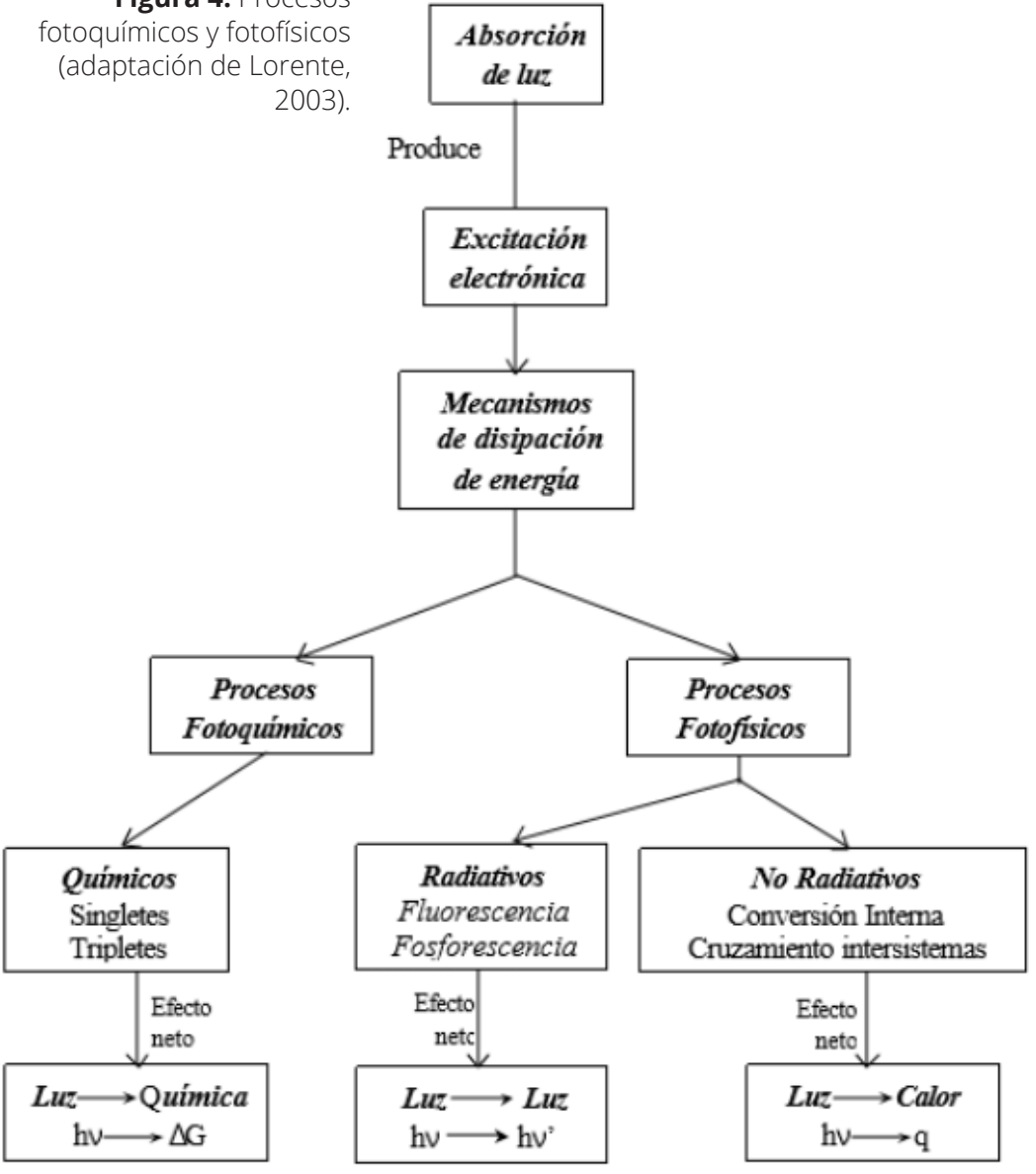

En realidad, hay muchas vías posibles de desexcitación, es decir, pérdida de energía tanto fotoquímica como fotofísica. En consecuencia, la más favorable dependerá del tipo de molécula y de la naturaleza de los estados excitados implicados.

Por otro lado, los procesos radiativos se distinguen de los procesos no radiativos porque los primeros implican la emisión de radiación electromagnética para ir desde el estado excitado hasta el estado fundamental (que es el nivel de menor energía en un átomo o molécula); mientras que en los procesos no radiativos, se puede pasar de un estado a otro sin ninguna emisión, dando como resultado efectos térmicos (ver figura 5).

\section{Clasificación por procesos fotoquímicos y fotofísicos}

Bajo condiciones ya mencionadas, cuando la radiación electromagnética se acerca a una molécula, se puede producir una alteración en las energías de enlace de la molécula. Esta alteración puede conducir a una reacción en la que la molécula absorbe la radiación y se produce un cambio en su estructura energética (organización de los niveles de energía molecular) (Gomez Lara, 2005).

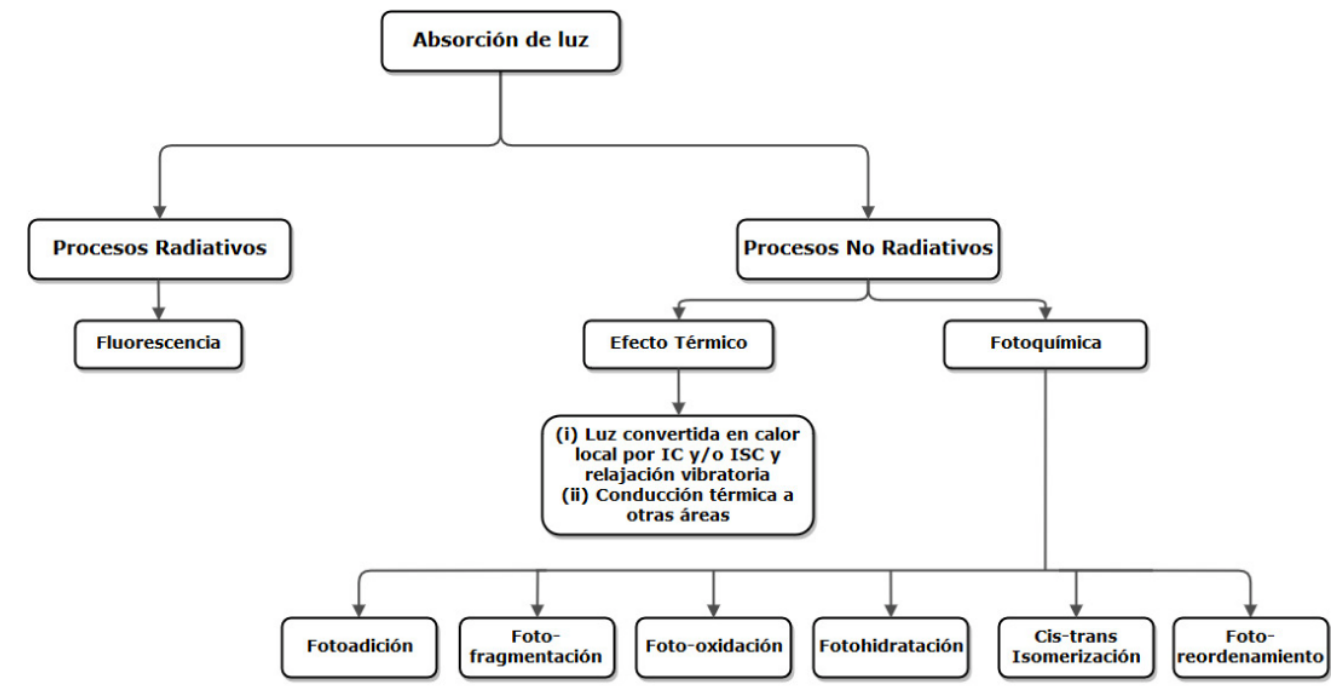

Figura 5. Clasificación por procesos radiativos y no radiativos (adaptación de Prasad, 2004). 


\section{Procesos fotoquímicos}

Un proceso fotoquímico es un fenómeno de naturaleza física que involucra la absorción de determinada radiación electromagnética por una molécula, lo que produce estados electrónicamente excitados de gran reactividad química (Lorente, 2003). Dicho de otra manera, cuando una molécula absorbe luz, ésta posee mayor energía, lo que se conoce como molécula excitada, dicha energía se puede perder cuando la molécula reacciona con otras, lo que genera una transformación de la materia. Una forma de que se produzcan las reacciones fotoquímicas es mediante la absorción de radiación electromagnética: ultravioleta (100-400 nm), visible (400-700 nm) o infrarroja (700-1000 nm) (Avery, 1982).

Un ejemplo de esta interacción se puede observar en la figura 6, en la cual se muestra la transformación de moléculas de dióxido de carbono y agua en glucosa y oxígeno, a través de un proceso fotoquímico (Atkins y de Paula, 2008).

\section{Procesos fotofísicos}

Por otra parte, un proceso fotofísico involucra sólo cambios en los estados de energía (estados cuánticos) de las moléculas, sin afectar su naturaleza química. Algunas de las formas físicas de desexcitación de las moléculas son la emisión de luz o transferencia de calor.

Figura 6. La fotosíntesis, un ejemplo esquemático de un proceso fotoquímico (adaptación de Blueringmedia, 2020).
En realidad, existen muchos procesos de desexcitación fotofísica, los cuales suelen ser muy rápidos y se pueden clasificar de la siguiente manera: radiativos y no radiativos.

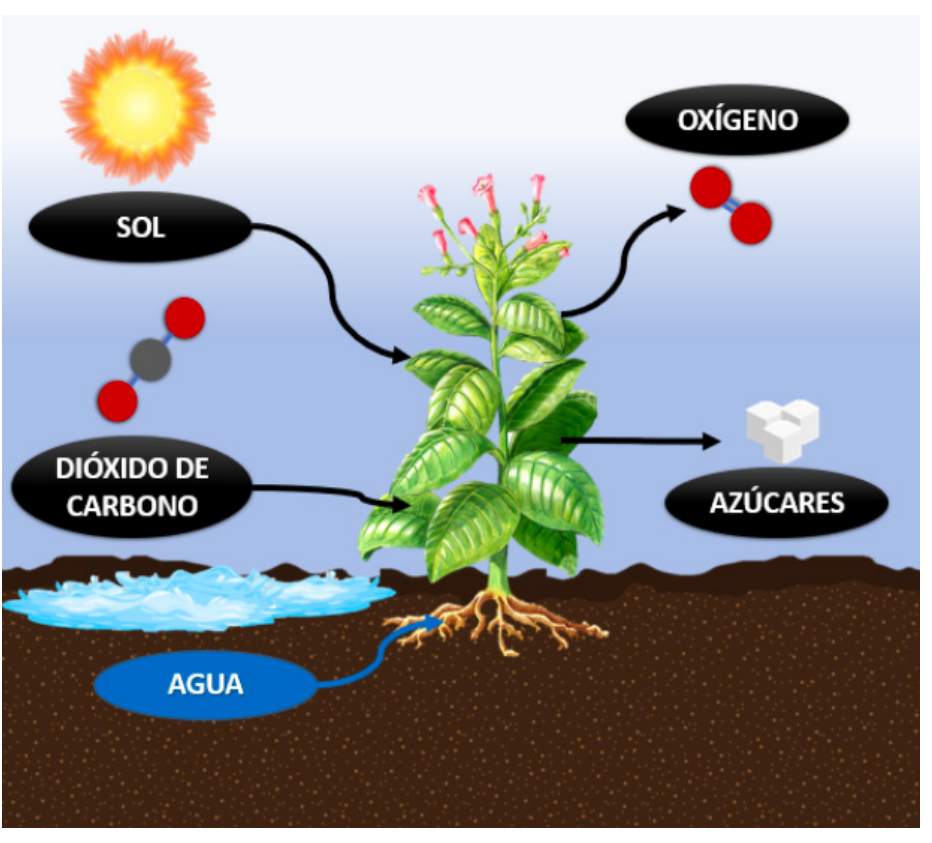

\section{Clasificación por procesos radiativos y no radiativos}

Por otro lado, podemos abordar los fenómenos que describen las interacciones moleculares con la luz, desde una perspectiva diferente. Este enfoque está dado principalmente por la biofotónica, ciencia encargada del uso de la luz en aplicaciones biológicas y médicas; por lo cual, está mayormente enfocada a moléculas orgánicas (ver figura 5).

Tomando en cuenta lo anterior, podemos clasificar las interacciones de la luz con dichas moléculas en dos caminos diferentes: Ios procesos radiativosy no radiativos. Estos procesos, como ya se mencionó anteriormente, se distinguen por tener diferentes caminos de desexcitación, ya sea por la emisión de luz (procesos radiativos) o sin necesidad de emitirla (procesos no radiativos). 


\section{Procesos radiativos}

Figura 7. Ejemplo de un proceso radiativo, donde se puede observar la fluorescencia y fosforescencia. Fuente: elaboración propia,
En los procesos radiativos, las moléculas excitadas reemiten radiación electromagnética, tendiendo, casi siempre, a regresar al estado energético fundamental, el de menor energía. Además, la cantidad de energía que es reemitida por las moléculas excitadas es menor que la energía que se utilizó para excitarlas.

\section{a) Iluminación con luz blanca.}

\section{b) Iluminación con luz ultravioleta (uv).}

\section{c) Desconectando la iluminación con luz uv.}

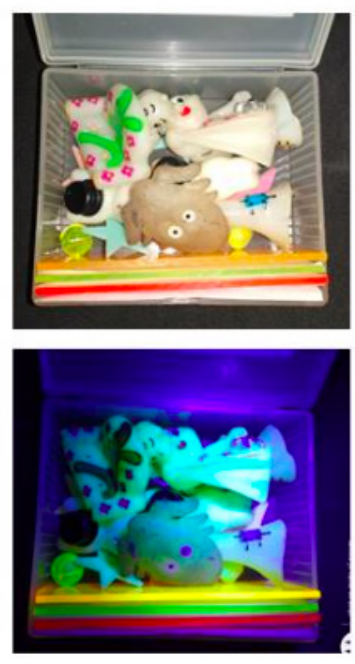

Emisión convencional

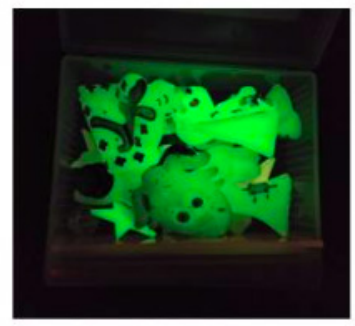

Solo se observa emisión fosforescente

Se observa emisión fosforescente $y$
Ejemplo de los procesos de emisión radiativos son la fluorescencia y la fosforescencia. En ambos fenómenos las moléculas que han absorbido radiación emiten luz. La fluorescencia es un fenómeno no muy común, pero la fosforescencia es aún menos frecuente en sistemas químicos. Este último fenómeno está confinado a compuestos químicos muy específicos y bajo condiciones extraordinarias. fluorescente

Una diferencia específica entre la fluorescencia y la fosforescencia es su tiempo de duración. La fluorescencia cesa de inmediato una vez que la fuente de excitación ha sido retirada de la sustancia (alrededor de $1 \times 10^{-6} \mathrm{~s}$, fracciones de segundos) mientras que la fosforescencia dura al menos unos $1 \times 10^{4} \mathrm{~s}$ (varios segundos) y en algunos casos hasta horas (Skoog, 1994; Atkins y de Paula, 2008), ejemplo de estos casos los podemos observar en la figura 7.

\section{Procesos no radiativos}

Cuando una molécula en estado excitado pasa a un estado diferente, de menor energía, pero sin emisión de radiación electromagnética, la transición, entonces, está dada por transferencia intramolecular de energía, es decir, que ocurre entre estados cuánticos (estados de energía) de una molécula, sin necesidad de perturbaciones externas como colisiones entre partículas. Se muestran ejemplos de estados vibracionales de moléculas (procesos no radiativos) en la figura 8. De esta manera, podemos encontrar dos tipos de desexcitaciones no radiativas: conversión interna y cruce intersistemas (Gomez Lara, 2005).

Aunque, estrictamente hablando, todas las desexcitaciones que no emiten radiación son no radiativas. Dichas transiciones dejan a la molécula con un exceso de energía vibracional. Pero esa energía puede ser removida rápidamente por colisiones o vibraciones moleculares, en un proceso denominado relajación vibracional (Lorente, 2003). 
Figura 8. Estados vibracionales de una molécula (procesos no radiativos). Adaptación de Nanofuturo, 2020.
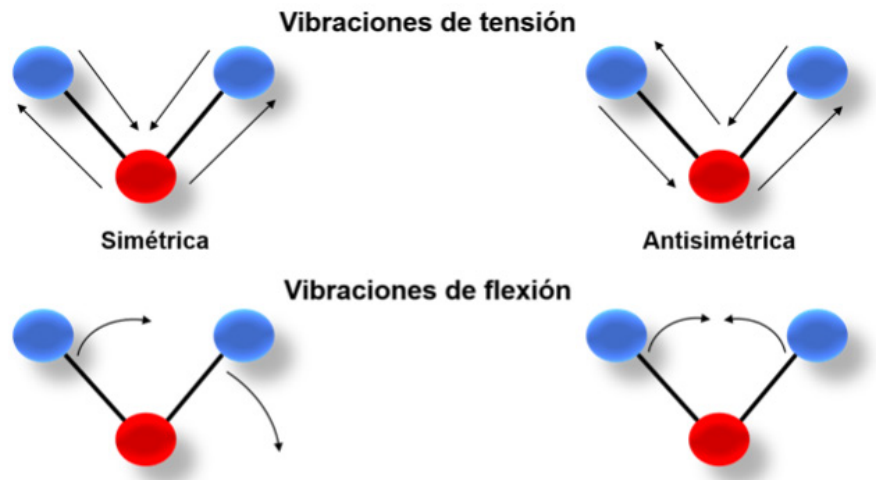

Balanceo en el plano
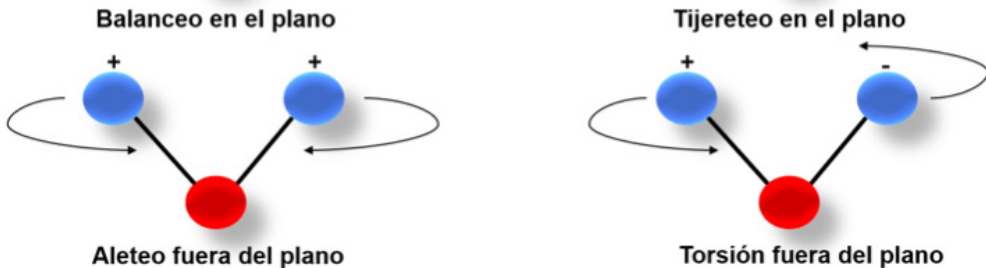

Esta forma de relajación es muy eficiente, prueba de ello es que la mayoría de las moléculas no son fluorescentes; además, que las bandas de emisión siempre se observan desplazadas hacia longitudes de ondas (del espectro electromagnético) más largas, respecto a las bandas de absorción inicial. Por lo tanto, el camino más propicio hacia el estado fundamental es aquel que minimiza el tiempo de vida del estado excitado (Prasad, 2004).

En lo que compete a los procesos radiativos, para el caso particular de componentes biológicos, encontramos fenómenos como fluorescencia $y$, en algunos casos, fosforescencia; mientras que de los procesos no radiativos se desprenden dos líneas que pueden seguir las moléculas para alcanzar un estado de menor energía (estado basal); éstas son: efectos térmicos y efectos fotoquímicos (Prasad, 2004, ver figura 5).

Los efectos térmicos resultan de la conversión de la energía de la luz a calor, a través de una combinación de procesos no radiativos como la conversión interna, el cruce intersistemas y relajaciones vibratorias, los cuales han sido explicados anteriormente.

Para el caso de la fotoquímica, la interacción de la luz con las moléculas origina una transformación química en ellas, principalmente por la absorción de radiación electromagnética de cierta longitud de onda. En estos procesos, no existe sólo una forma de transformación, ya que eso depende tanto de las moléculas como de la excitación en ellas; es así como podemos encontrar diferentes tipos de reacciones, denominadas reacciones fotoquímicas, debido a la importancia de la luz en dichos procesos. 


\section{Conclusión}

Este trabajo se reconoce la clasificación de los procesos de interacción luzmateria y el entendimiento de cada uno de los fenómenos que se presentan. Como se describió, los efectos fotofísicos y fotoquímicos están presentes en muchos de los fenómenos que conocemos. Además, se puede comprender cómo estos mecanismos están estrechamente ligados, y que en algunos casos la presencia de alguno de ellos lleva a la aparición de otro.

Además, vemos que las clasificaciones pueden tener diferentes enfoques. Por una parte, se hace la distinción entre procesos físicos y químicos relacionados con radiación; mientras que, por otra parte, se abordan los fenómenos a partir de la diferenciación entre aquellos que emiten luz y los que no lo hacen.

Uno de los aspectos importantes a resaltar es la relevancia de los procesos fotofísicos y fotoquímicos, presentes hoy en día, pues éstos pueden ser ampliamente aplicados en distintas áreas: síntesis química, biología, medicina y tecnologías. Por ejemplo, la fotoquímica es ampliamente utilizada en la síntesis de compuestos químicos, especialmente en química fina, también en la degradación de compuestos contaminantes, que pueden ser de grado farmacéutico u orgánico, en superficies o fluidos, y en la producción de microorganismos con fotobiorreactores.

Por otro lado, los procesos fotofísicos, como la fluorescencia y fosforescencia, son aplicados en el campo de la medicina y biología; como desarrollo de quimiosensores, capaces de detectar y analizar moléculas vitales en sistemas vivos; o de importancia ambiental, por ejemplo, como apoyo en la detección de enfermedades como diabetes o cáncer.

Un comentario final importante es que no hay que confundir los procesos descritos en este artículo, con aquellos de la bioluminiscencia. La bioluminiscencia que se observa por ejemplo en las luciérnagas, hongos, bacterias y peces, se lleva a cabo por un proceso puramente bioquímico; en la bioluminiscencia se emite luz por una reacción química que se lleva a cabo, es una conversión de energía química a luminosa.

\section{Referencias}

* Atkins, P. y de Paula, J. (2008). Química Física. Médica Panamericana.

* Avery, H. E. (1982). Cinética química básica y mecanismos de reacción. Reverté S.A.

* Blueringmedia. (2020). Diagram showing process of photosynthesis. Adobe Stock. https://adobe.ly/2E25pnN

* Chemical Bonding: Chemical Formulas. (2020). Free Leaving Cert Notes. http:// leaving-cert-notes.weebly.com/chemical-bonding.html 
"La interacción de la luz con moléculas" Bárbara Paulet Domínguez Capitaine, Héctor Hugo Cerecedo Núñez, Patricia Padilla Sosa y Josué Ismael García Ramírez Vol. 21, Núm. 5, septiembre-octubre 2020 Revista Digital Universitaria

* Cruz-Garritz, D., Chamizo, J. A., y Garritz, A. (1991). Estructura Atomica. Un enfoque Químico. Addison-Wesley Iberoamericana.

* De la Rosa Acosta, M. Á. (2005). La luz en biología: Aplicaciones de interés agrícola e industrial. Universidad de Sevilla.

- Gela Irualia [Ikasi Etxean y Egin Gelan]. (2017, 10 de abril). FISIKA ETA KIMIKA. https:// sites.google.com/site/dbhfisikaetakimika/3-dbh/3-gaia-atomoa

* Gomez Lara, I. (2005). Estudio AB initio de mecanismos de reacción en sistemas moleculares fotosensibles [tesis doctoral, Universitat Rovira i Virgili]. Tesis Doctorals en Xarxa. https://www.tdx.cat/handle/10803/9075\#page=1

- Lorente, C. (2003). Fotofísica y propiedades fotosensibilizadoras de pterinas en solución acuosa (tesis doctoral, Facultad de Ciencias Exactas, Argentina). Repositorio institucional de la unLP. http://sedici.unlp.edu.ar/handle/10915/2216

* Nanofuturo. (2020). Espectroscopía TF-IR. https://nanocienciainforma.wordpress. com/espectroscopia-tf-ir/

* Ronan, P. (2017). EM spectrum.svg. [espectro de las ondas electromagnéticas]. https://es.m.wikipedia.org/wiki/Archivo:EM_spectrum_es.svg

* Prasad, P. N. (2004). Introduction to Biophotonics. John Wiley \& Sons, Inc.

* Wikipedia. (2020). Bioluminiscencia. https://es.wikipedia.org/wiki/Bioluminiscencia

* Xu Panda. (2017, 6 de abril). HeNe Laser Levels.png [Energy levels in a He-Ne Laser]. https://en.wikipedia.org/wiki/Helium\%E2\%80\%93neon_laser\#/media/File:HeNe_ Laser_Levels.png

\section{Cómo CITAR ESTE ARTículo}

- Domínguez Capitaine, Bárbara Paulet, Cerecedo Núñez, Héctor Hugo, Padilla Sosa, Patricia y García Ramírez, Josué Ismael. (2020, septiembre-octubre). La interacción de la luz con moléculas. Revista Digital Universitaria (RDU), 21(5). DOI: http://doi.org/10.22201/cuaieed.16076079e.2020.21.5.1 